\title{
Amelioration of collagen-induced arthritis by thrombin inhibition
}

\author{
Ingrid Marty, Veronique Péclat, Gailute Kirdaite, Roberto Salvi, Alexander So, \\ and Nathalie Busso \\ Laboratoire de Rhumatologie, Centre Hospitalier Universitaire Vaudois, Lausanne, Switzerland \\ Address correspondence to: Nathalie Busso, Centre Hospitalier Universitaire Vaudois, \\ Laboratoire de Rhumatologie, Nestlé 05-5029, 1011 Lausanne, Switzerland. \\ Phone: 41-21-314-1443; Fax: 41-21-314-1533; E-mail: Nathalie.Busso@chuv.hospvd.ch.
}

Received for publication August 15, 2000, and accepted in revised form January 23, 2001.

\begin{abstract}
The deleterious role of fibrin deposition in arthritic joints prompted us to explore the effect of the thrombin inhibition on the course of collagen-induced arthritis (CIA) in the mouse. CIA was induced in male DBA/ $1 \mathrm{~J}$ mice using native chicken type II collagen. The thrombin inhibitor polyethyleneglycol-hirudin (PEG-hirudin) was given for 16 days, starting 20 days after the first immunization (preventive treatment) or at the onset of clinical signs of arthritis (curative treatment). All the mice treated with PEG-hirudin had a significantly prolonged clotting time compared with control mice. PEG-hirudin, administered in a preventive way, led to significantly reduced incidence and severity of CIA during most of the treatment period, as assessed by clinical scoring. Accordingly, histological features showed a significant diminution of synovial hyperplasia in PEG-hirudin-treated mice compared with untreated mice. There was also a significant downmodulation of the synovial proinflammatory IL-1 $\beta$ and IL-12p35 cytokine mRNAs in treated mice. Intra-articular fibrin, evaluated by immunohistochemistry, was significantly reduced in treated mice compared with control mice and correlated with both clinical and histological scorings. Most importantly, once arthritis was established, PEG-hirudin also showed a curative effect. In conclusion, PEG-hirudin can both prevent the onset of CIA in a dose-dependent manner and ameliorate established arthritis, suggesting that thrombin inhibition may offer a new therapeutic approach in arthritis.
\end{abstract}

J. Clin. Invest. 107:631-640 (2001).

\section{Introduction}

Rheumatoid arthritis (RA) is a chronic inflammatory disease characterized by synovial inflammation and hyperplasia leading to progressive cartilage and bone destruction (1). Current concepts on the pathogenesis of RA invoke a $\mathrm{T}$ cell-driven process in synovitis initiation (2), but this mechanism may not be necessary to sustain chronic inflammation. Among other potential mechanisms, activation of the coagulation cascade in RA joints may represent one important nonimmunological pathway amplifying and perpetuating synovitis.

Extravascular coagulation may be initiated by local immunological and inflammatory stimuli that induce tissue factor (TF) expression (3). TF-mediated extrinsic coagulation pathway involves the sequential activity of the serine proteases factor VIIa, factor Xa, and thrombin, the latter enzyme converting fibrinogen into fibrin. Evidence supporting ongoing coagulation within the rheumatoid joint include the demonstration of an intact coagulation pathway and the existence of increased expression of TF and thrombin within the synovium $(4,5)$. Moreover, in RA synovial fluids, reduced levels of coagulation factors with concomitantly increased concentrations of thrombin activity and thrombin-antithrombin (TAT) complexes have been found, reflecting activation of the coagulation cascade $(6,7)$. Finally, the abundant extravascular fibrin deposition within the arthritic joints, which represents one of the most striking pathologic features of this disease in both human and experimental RA, provides a definitive proof for ongoing coagulation $(8,9)$.

Interactions between coagulation and inflammation are based on several observations. First, some coagulation factors, besides their well-known role in the coagulation cascade, have been shown recently to have direct cellular effects (10). Indeed, the circulating serine proteases factor VIIa, factor $\mathrm{Xa}$, and thrombin, upon binding to their cognate receptors TF (for factor VIIa), a cellular-binding site for factor Xa and proteinase-activated receptor-1 (PAR-1) for thrombin, induce proinflammatory responses both in vitro and in vivo. On the other hand, fibrin, the end-product of the coagulation cascade, has proinflammatory effects. Extravascular fibrin meshwork can serve as a matrix onto which inflammatory cells can adhere and migrate. Fibrin and fibrin(ogen) degradation products may also have a role by increasing vascular permeability and inducing chemotaxis at inflammatory sites $(11,12)$ and by enhancing monocyte expression of IL-1 13 , IL-6 (13, 14), and endothelial cell IL-8 production (15).

The role of intra-articular fibrin, whose formation depends on the balance between coagulation and fibri- 
nolysis, has been explored in experimental arthritis. We have demonstrated previously in the antigen-induced arthritis (AIA) model, that mice deficient in urokinasetype plasminogen activator (uPA) or plasminogen had impaired fibrinolysis that resulted in fibrin persistence and that exacerbated arthritis (16). In the same experimental model, the severity of arthritis was attenuated by defibrinogenation with ancrod (16) or by the genetic deficiency of fibrinogen (N. Busso, unpublished results). Taken together, these results stress the detrimental role of intra-articular fibrin deposition in arthritis, but the presence of residual inflammation in ancrod-treated or fibrinogen-deficient mice suggests that fibrin-independent effects may also occur.

Thrombin can play a role in sustaining joint inflammation during RA, based on several in vitro observations. Thrombin can promote the chemotaxis of neutrophils and monocytes and their adhesion to endothelial cells. It can also induce synovial cell proliferation $(17,18)$ and can act on endothelial cells to increase vascular permeability and the synthesis of various proinflammatory molecules (reviewed in ref. 19). These cellular events of thrombin are mediated by thrombin receptors, of which three are recognized, PAR-1, PAR-3, and PAR-4 (20). Of these, the PAR-1 thrombin receptor is abundantly expressed in inflamed rheumatoid synovial tissues (21). PAR-1 is a member of the family of G-coupled proteins with seven transmembrane domains (22). This multifunctional receptor is activated by proteolytic cleavage of an $\mathrm{NH}_{2}$ terminus extra-membrane fragment by thrombin such that the newly created $\mathrm{NH}_{2}$ terminus can then act as a "tethered ligand" leading to receptor activation (20). PAR-1 activation can be blocked by hirudin, a highly specific noncovalent thrombin inhibitor produced by the salivary glands of the leech Hirudo medicinalis (23). One of the limiting factors for its therapeutic use is its rapid elimination from the circulation with a plasmatic half-life of about 1 hour (24). Polyethyleneglycolhirudin (PEG-hirudin), a recombinant hirudin covalently bound to two PEG-5,000 residues exhibits a significantly prolonged half-life (8-9 hour) with no loss of potency or specificity $(25,26)$.

Little is known about the in vivo effects of anticoagulants, in particular of thrombin inhibitors, on arthritis. We decided, therefore, to evaluate the effect of PEGhirudin on collagen-induced arthritis (CIA) in mice. CIA is a well-established experimental model of RA that can be induced in susceptible strains of mice by immunization with native heterologous type II collagen (27). Both humoral and cellular immunity are implicated in this model, because anticollagen type II Ab and collagen type II-specific Th1 cells are necessary for the development of arthritis (28). The resulting disease is a chronic proliferative synovitis with important cartilage destruction, bone erosion, and periosteal bone formation leading later to joint alterations and significant deformities, thus sharing many histopathologic features with human RA. We hypothesized that thrombin inhibition could have beneficial effects in an experimental model of arthritis by reducing intra-articular fibrin levels and by blocking PAR-1-mediated proinflammatory effects.

\section{Methods}

Induction of arthritis. Male DBA/1J mice between 8 and 10 weeks of age were obtained from BRL/RCC Biotechnology \& Animal Breeding (Füllinsdorf, Switzerland). Native chicken type II collagen (Chondrex, Redmond, Washington, USA) was dissolved in $0.1 \mathrm{M}$ acetic acid overnight at $2 \mathrm{mg} / \mathrm{ml}$. Collagen $(100 \mu \mathrm{g})$ emulsified in CFA containing $5 \mathrm{mg} / \mathrm{ml}$ tuberculosis mycobacterium was injected intradermally at the base of the tail. At day 24 after the first injection, a booster injection of $100 \mu \mathrm{g}$ of native chicken collagen was given in incomplete Freund's adjuvant intradermally at the base of the tail. All immunization reagents were purchased from DIFCO (Basel, Switzerland). Institutional approval was obtained for these experiments.

Clinical scoring. Mice were carefully examined each day from day 18 after the first collagen injection for the visual appearance of arthritis in peripheral joints. The clinical severity of arthritis was scored as follows: 1 point for each swollen digit except the thumb (maximum, 4), 1 point for the tarsal or carpal joint, and 1 point for the metatarsal or metacarpal joint with a maximum score of 6 for a hindpaw and 5 for a forepaw. Each paw was graded individually, the cumulative clinical arthritic score per mouse reaching a maximum of 22 points.

Histological grading of arthritis. At least 12 mice per group were sacrificed, and the knees were dissected and fixed in $10 \%$ buffered formalin for 7 days. Fixed tissues were decalcified for 3 weeks in 15\% EDTA, dehydrated, and embedded in paraffin. Sagittal sections $(8 \mu \mathrm{m})$ of the whole knee joint were stained with Safranin-O and counterstained with fast green/iron hematoxylin. Histological sections were graded independently by two observers unaware of animal treatment using an established scoring system for synovial hyperplasia (from 0 , no hyperplasia, to 3 , most severe hyperplasia) and inflammatory cells in synovium ( 0 , no inflammation, to 3 , severely inflamed joint). Cartilage damage was determined by Safranin-O staining (from 0, no change from normal nonarthritic knee joint, fully stained cartilage, to 3, total loss of Safranin-O staining). For each group, paws of some mice were also dissected and processed for histological staining as described above.

Fibrin immunohistochemistry. Paraffin-embedded sections were deparaffinized and rehydrated, then incubated for 30 minutes at room temperature with 5\% BSA and $20 \%$ normal goat serum. Endogenous peroxidase activity was blocked with $3 \% \mathrm{H}_{2} \mathrm{O}_{2}$ for 10 minutes. Slides were then overlayed with rabbit anti-mouse fibrinogen serum (generously provided by Jay Degen, Children's Hospital Research Foundation, Cincinnati, Ohio, USA), diluted 1:1000, for 30 minutes at room temperature.

Bound $\mathrm{Ab}$ was visualized using the avidin-biotin-peroxidase complex (Vectastain Elite ABC kit; Vector Laboratories, Burlingame, California, USA). The color was 
developed by 3,3'-diaminobenzidine (Sigma Chemical Co., Basel, Switzerland) containing $0.01 \% \mathrm{H}_{2} \mathrm{O}_{2}$. After extensive washing in water, slides were counterstained with Papanicolaou and mounted in Merckoglass. Staining specificity was confirmed using, as primary Ab's, nonimmune rabbit serum or fibrinogen-preadsorbed immune serum. An incubation without the first $\mathrm{Ab}$ served as a negative control. Fibrin immunostaining in the knee joints was graded independently by two observers unaware of animal treatment, on a scale of 0 (no fibrin at all) to 6 (maximum fibrin staining).

PEG-hirudin treatment. PEG-hirudin (Knoll AG-BASF Pharma, Ludwigshafen, Germany) was used. The solution was reconstituted from the supplied powder at a concentration of $0.1 \mathrm{mg} / \mathrm{ml}$ in PBS. The mice were injected subcutaneously with $200 \mu \mathrm{l}$ of this solution or a tenfold dilution of this solution once per day (final concentration of PEG-hirudin $1 \mathrm{mg} / \mathrm{kg}$ and $0.1 \mathrm{mg} / \mathrm{kg}$, respectively). The treatment was started at day 20 (i.e., 3 days before the boost injection) for the study of the preventive effect or at the appearance of the first clinical symptoms (clinical scoring greater than or equal to 1) for the study of the curative effect.

Clotting times. To obtain plasma, blood was collected from the tail vein or from the inferior vena cava in anesthetized animals in $0.12 \mathrm{M}$ trisodium citrate ( $1 \mathrm{vol}$. of citrate to 9 vol. of blood). Blood samples were centrifuged at $1,500 \mathrm{~g}$ for 15 minutes at $4^{\circ} \mathrm{C}$ and plasmas stored at $-20^{\circ} \mathrm{C}$ until use. All clotting times, performed on citrated plasma diluted in Owren's buffer (sodium diethylbarbiturate buffer, $\mathrm{pH} 7.35$ ) prewarmed at $37^{\circ} \mathrm{C}$, were calculated using an Amelung micro-coagulometer (Dialine, Itingen, Switzerland). For determination of the prothrombin time (PT), $50 \mu \mathrm{l}$ of fivefold diluted plasma were used. After addition of $100 \mu \mathrm{l}$ of a thromboplastin reagent (Recombiplastin Ortho; Almedica, Galmiz, Switzerland), time to thrombus formation was recorded. For activated partial thrombin time (aPTT) $50 \mu \mathrm{l}$ of a twofold diluted plasma was incubated with $50 \mu \mathrm{l}$ of activated cephalin reagent (Actin; DadeBehring, Marburg, Germany) for 2 minutes at $37^{\circ} \mathrm{C}$. After addition of $50 \mu \mathrm{l}$ of $0.025 \mathrm{M} \mathrm{CaCl}_{2}$, time to thrombus formation was recorded. For thrombin time (TT) measurement, $50 \mu \mathrm{l}$ of twofold diluted plasma was used. After addition of $50 \mu \mathrm{l}$ of a twofold diluted thrombin reagent (Thromboclotin; Dade-Behring), time to thrombus formation was recorded.

TAT determinations. TAT complex concentration in plasma was measured by a commercially available ELISA kit designed for human TAT (Enzygnost TAT; Dade-Behring), which also cross-reacts with murine TAT. The content of murine TAT in plasma was calculated according to the human TAT standard curve.

Tissue extracts preparation. Tissue extracts were prepared from cryostat sections of paws as described previously (16).

RNase protection assay. Cryostat sections of murine synovial tissues from knee joints were homogenized in Trizol reagent (GIBCO BRL, Basel, Switzerland), and total RNA extractions were performed according to the manufacturer's instructions. RNase protection assays were performed using a standard procedure (29). The antisense probe set, including PAR-1 and GAPDH, was produced by T7 RNA polymerase-driven transcription of the corresponding cDNA plasmids (30). For cytokine mRNA measurements, the mCK-2 multiprobe set was used (PharMingen, Basel, Switzerland). PhosphorImager analysis was performed to quantify mRNA levels of each gene.

Anticollagen Ab's measurement. Maxisorp-Nunc 96-well plates (Life Technologies AG, Basel, Switzerland) were coated overnight at $4^{\circ} \mathrm{C}$ with $5 \mu \mathrm{g} / \mathrm{ml}$ of chicken type II collagen in PBS and blocked for 8 hours at $4^{\circ} \mathrm{C}$ with $1 \% \mathrm{BSA}$ in PBS containing $200 \mathrm{mM} \mathrm{NaCl}$ and $0.05 \%$ Tween-20 (BSA/PNT). One hundred microliters of plasma, diluted 1:100 in BSA/PNT and $100 \mu \mathrm{l}$ of serially diluted $\mathrm{mAb}$ against broadly reactive conserved epitopes of native collagen type II (used to construct a standard curve) were incubated overnight at $4^{\circ} \mathrm{C}$. Wells were washed four times with PNT. Then, 100 $\mu \mathrm{l} /$ well of alkaline phosphatase conjugated antimouse IgG (Sigma Chemical Co.) diluted 1:500 in $0.05 \%$ Tween-20, $50 \mathrm{mM}$ Tris- $\mathrm{HCl}$, and $140 \mathrm{mM} \mathrm{NaCl}$ (TTBS) was added. After washing four times with TTBS, color was developed with $100 \mu \mathrm{l} /$ well of $p$-nitrophenylphosphate (Sigma Chemical Co.) and the reaction stopped by adding $50 \mu \mathrm{l} /$ well of $3 \mathrm{M} \mathrm{NaOH}$. Plate reading was performed at $450 \mathrm{~nm}$.

Statistical analysis. The Wilcoxon/Kruskal-Wallis (rank sum test) for unpaired variable (two-tailed) with nonGaussian distribution or the unpaired Student's $t$ test for normally distributed variables was used to compare differences between groups. To evaluate the strength of the relationship between variables, the univariate correlation was used. $P$ values less than 0.05 were considered significant.

\section{Results}

Synovial coagulation and thrombin generation during CIA

Substantial fibrin deposits, as detected by immunohistochemistry, were present in arthritic knee and paw joints of mice with CIA within the synovium, in the synovial cavity, and on the surface of the articular cartilage (Figure 1, b and $\mathrm{f}$ ), indicating the presence of coagulation. The specificity of the antiserum was confirmed by the absence of signal obtained with normal rabbit serum or with immune serum preincubated with an excess of purified murine fibrinogen (results not shown). Ongoing activation of the coagulation cascade was further demonstrated by increased TAT levels in synovial tissue extracts from arthritic mice compared with the undetectable levels in naive mice (Table 1). Increased levels of TAT were also found in the plasma of CIA mice compared with naive mice, reflecting the overall increased level of thrombin generation (Table 1). Finally, the synovial expression of the thrombin 

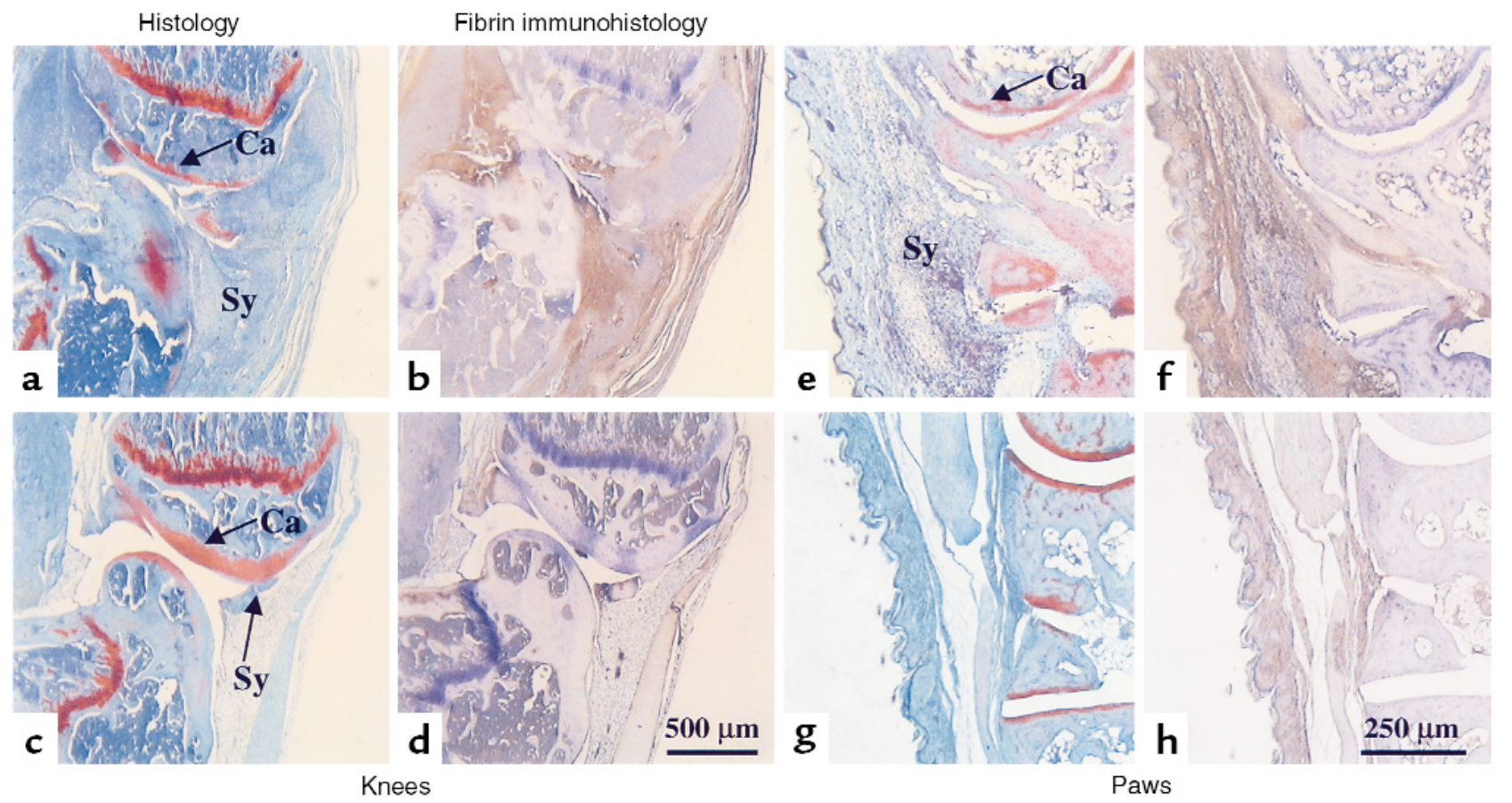

Figure 1

Histologies and immunohistologies of joints from control and PEG-hirudin-treated mice with $\mathrm{CIA}$. (a and $\mathbf{c})$ Safranin-O-stained sections of control and PEG-hirudin-treated knee joints at day 36 after immunization. Note the size difference of synovial membrane (Sy), which is thicker in the control arthritic mice (a) than in the PEG-hirudin-treated mice (c). Red proteoglycan staining of the articular cartilage matrix (Ca) is decreased in the control group. (b and $\mathbf{d}$ ) Fibrin immunostaining of adjacent sections using a rabbit anti-murine fibrin(ogen) Ab. Brown color indicates positivity. (e and $\mathbf{g}$ ) Safranin-O-stained sections of control and PEG-hirudin-treated paw joints at day 36 after arthritis induction. Note the difference in thickness of the synovial membrane, which is thicker in the control arthritic mice (e) than in the PEG-hirudin-treated one. ( $\mathbf{f}$ and $\mathbf{h}$ ) Fibrin immunostaining of adjacent sections.

receptor PAR-1, as estimated by its mRNA levels, was increased approximately twofold in CIA joints compared with naive ones (Table 1).

\section{Anticoagulant activity of PEG-hirudin}

Effect on coagulation times. From preliminary experiments, we established that a single injection of PEG-hirudin at either $0.1 \mathrm{mg} / \mathrm{kg}$ or $1 \mathrm{mg} / \mathrm{kg}$ led to a significant prolongation of TT ( $76 \pm 25$ seconds for the low dose, more than 250 seconds for the high dose, 5 hours after PEGhirudin administration); for both doses, TT remained significantly prolonged over 20 hours, although the effect was more pronounced with the high dose (data not shown). We next measured the effect of PEGhirudin treatment (once-daily injection of PEG-hirudin at $1 \mathrm{mg} / \mathrm{kg}$ for 16 days) on aPTT, $\mathrm{TT}$, and PT in mice with CIA (Table 2). The aPTT and TT were significantly prolonged in treated mice compared with placebotreated mice $(P<0.001)$. No modification of the PT was observed with this treatment.

Effect on TAT complexes. Because each new thrombin molecule formed in plasma will immediately be coupled to ATIII, TAT com-

\section{Table 1}

plexes were measured as a surrogate for the circulating thrombin levels. We compared plasma TAT levels of placebo and PEG-hirudin-treated mice (Table 2). There was a marked decrease in plasma TAT levels in anticoagulated versus untreated CIA mice $(9.99 \mathrm{ng} / \mathrm{ml}+3.24$ vs. $59.7 \mathrm{ng} / \mathrm{ml}+15.75 ; P<0.01)$.

\section{Effect of PEG-hirudin on CIA incidence and severity}

In our study, DBA-1 mice developed a gradual onset of CIA at day 20 after immunization, and the incidence reaches more than $80 \%$ by day 40 . To investigate the effect of PEG-hirudin on CIA onset, mice at day 20 after immunization were injected daily with $1 \mathrm{mg} / \mathrm{kg}$ of PEG-hirudin $(n=13)$ or with PBS $(n=13)$. Treatment lasted for 16 days until sacrifice at day 36 . Animals were

TAT measurements and PAR-1 mRNA levels of naive and CIA mice

\begin{tabular}{lccc}
\hline & Tissue TAT & Plasma TAT & Synovial tissue PAR-1 mRNA \\
Naive mice & Not detectable, $n=4$ & $17.2 \pm 7.9, n=7$ & $100 \pm 37.5, n=4$ \\
Mice with CIA & $0.71 \pm 0.27, n=6^{\mathrm{A}}$ & $59.7 \pm 15.8, n=13^{\mathrm{A}}$ & $224 \pm 33, n=4^{\mathrm{A}}$ \\
\hline
\end{tabular}

Tissue and plasma TAT levels were measured in mice without CIA (naive mice) as well as in mice with CIA at day 36 . For this purpose, citrated plasma and tissue extracts prepared from total paws were collected. For PAR-1 mRNA determination, total RNA was prepared from knee joints of naive or from arthritic CIA knee joints. Expression of PAR-1 mRNA was determined by RNase protection assay and Phosphorlmager analysis. Comparison of mRNA levels was done after standardization with GAPDH mRNA. The results are expressed as mean + SEM. ${ }^{A} P<0.05$. 
Table 2

Coagulation times and plasma TAT levels of placebo and PEG-hirudin-treated CIA mice

\begin{tabular}{lcccc}
\hline & aPTT $(\mathrm{s})$ & Thrombin time $(\mathrm{s})$ & Prothrombin time $(\mathrm{s})$ & Plasma TAT $(\mathrm{ng} / \mathrm{ml})$ \\
Mice with CIA & $28.85 \pm 1.14, n=10$ & $30.67 \pm 1.01, n=14$ & $12.80 \pm 1.35, n=14$ & $59.7 \pm 15.8, n=13$ \\
Mice with CIA + PEG-hirudin & $44.20 \pm 3.26^{\mathrm{A}}, n=14$ & $>250 \pm 0^{\mathrm{A}}, n=14$ & $11.71 \pm 1.32, n=14$ & $9.9 \pm 3.24^{\mathrm{B}}, n=13$
\end{tabular}

aPTT, TT, PT, and plasma TAT levels were measured in untreated and PEG -hirudin-treated mice with CIA (treatment with $1 \mathrm{mg} / \mathrm{kg} / \mathrm{day}$ PEG-hirudin subcutaneously, from day 20 to day 36). Citrated plasmas were prepared at day 36 from blood collected between 1 and 2.5 hours after the last injection of PEGhirudin. The results are expressed as mean \pm SEM. Statistical significance was determined using the $t$ test. ${ }^{A} P<0.001 .{ }^{B} P<0.01$.

monitored daily by visual scoring for the appearance of clinical signs of arthritis. Treatment with PEG-hirudin $(1 \mathrm{mg} / \mathrm{kg}$ ) led to decreased disease incidence (Figure 2, $a$ and d) and arthritis severity (Figure 2, b and e). These effects were observed in the first days after initiation of treatment (Figure 2, a and b, a significant difference between the two treatment groups was found from day 24 to day 36). We also found a reduction in the number of affected paws in the PEG-hirudin-treated group (Figure 2c). The preventive effect of PEG-hirudin on CIA onset was efficient at both 0.1 and $1 \mathrm{mg} / \mathrm{kg}$; however, we observed a slightly reduced efficiency at 0.1 $\mathrm{mg} / \mathrm{kg}$ (Figure 2, $\mathrm{d}$ and $\mathrm{e}$ ).

\section{Effect of PEG-hirudin on established CIA}

The effect of PEG-hirudin on established CIA was also investigated (Figure 3). Mice were treated with daily injections of PEG-hirudin at $1 \mathrm{mg} / \mathrm{kg}(n=26)$ or with PBS $(n=28)$ for 16 days. Injections were commenced on the first appearance of clinical signs of arthritis (considered day 0 of clinical arthritis). There was a marked decrease in arthritis severity as assessed by clinical scores in PEG-hirudin-treated mice, which was significant both at the beginning and at the end of the treatment (Figure 3a). Moreover, at the end of the treatment (day 15 and 16) the number of affected paws was significantly reduced (Figure $3 \mathrm{~b}$ ). Taken together, these results demonstrate that PEG-hirudin, at the dose of 1 $\mathrm{mg} / \mathrm{kg} /$ day, had a curative effect on CIA.

\section{Figure 2}

Development of CIA in PEG-hirudin-treated mice. (a-c) From day 20 after type II collagen immunization, test mice were treated daily with PEG-hirudin (subcutaneous injection at $1 \mathrm{mg} / \mathrm{kg} / \mathrm{day}, n=13$ ). Placebo mice received PBS $(n=13)$. Mice were observed daily for development of arthritis. (a) Percentage of mice that developed arthritis. (b) Arthritis severity. Mean arthritic index + SEM is shown. (c) Number of arthritic paws (mean + SEM). Dose-response effect of PEGhirudin on incidence (d) and severity (e). From day 20 after immunization, test mice were treated daily subcutaneously with PEG-hirudin ( $0.1 \mathrm{mg} / \mathrm{kg}$ or $1 \mathrm{mg} / \mathrm{kg} ; n=13$ in each group). Placebo mice received PBS only $(n=13)$. Mice groups were compared by statistical analysis using the nonparametric Wilcoxon/Kruskal-Wallis tests (rank sums). (a) $P<0.05$ from day 24 to day 36 . (b) $P<0.01$ from day $24-27$ and $P<0.05$ until day 36 . (c) $P<0.05$ from day 24 to day 36. (d) $P<0.02$ from day 21 until day 27 for the dose of 0.1 $\mathrm{mg} / \mathrm{kg} . P<0.02$ from day 21 until day 28 and $P<0.05$ from day 28-31 for the dose of $1 \mathrm{mg} / \mathrm{kg}$. (e) $P<0.02$ from day 21 until day 27 for the dose of $0.1 \mathrm{mg} / \mathrm{kg}$ and until day 32 for the dose of $1 \mathrm{mg} / \mathrm{kg}$.
Synovial histological changes due to PEG-hirudin treatment

To determine whether the clinically observed effect of PEG-hirudin was associated with specific histopathological changes, we compared arthritic knee and paw joint histologies between placebo and treated mice. In the placebo group, the synovial membrane (noted Sy on figure) was significantly thicker than in PEGhirudin-treated animals (compare Figure 1, a and c, for knees and Figure 1, e and g, for paws). An effect on the articular cartilage was also observed, with a decreased loss of Safranin-O staining in the treated group (compare Figure 1, a and c, for knees and Figure 1 , e and g, for paws). A semiquantitative histological evaluation was performed on the knee sections using a four-point (0-3) scoring system to evaluate synovial hyperplasia, inflammatory cells in the synovial mem-

\section{a}

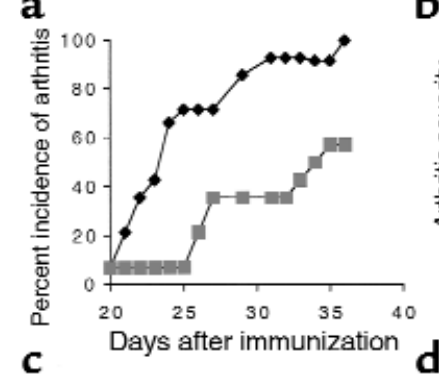

b

C
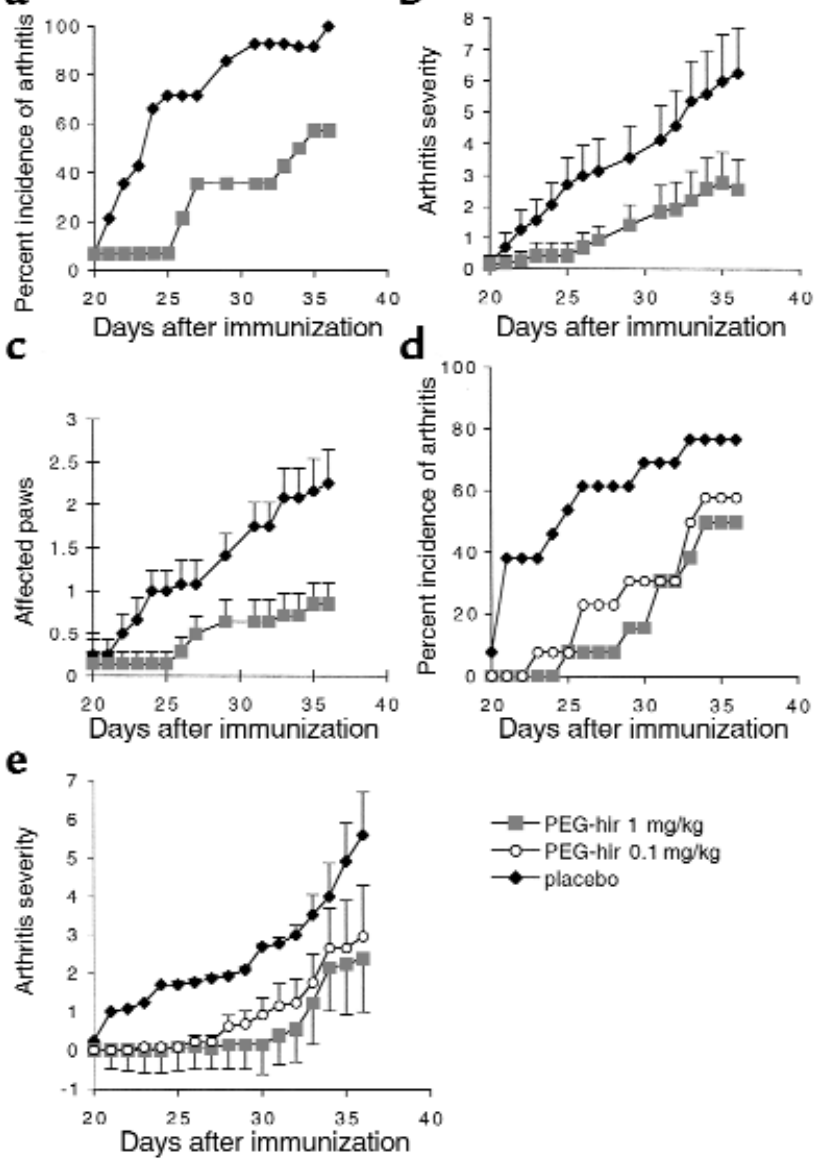

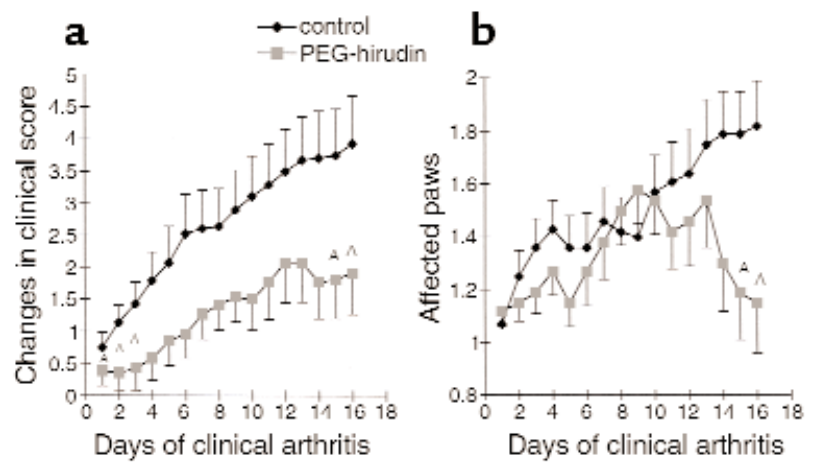

Figure 3

PEG-hirudin treatment of established arthritis. Test mice $(n=26)$ were treated subcutaneously daily with $1 \mathrm{mg} / \mathrm{kg}$ of PEG-hirudin from the first day of clinical arthritis for 16 days. Placebo mice $(n=28)$ received PBS. Groups of animals were compared by statistical analysis using the nonparametric Wilcoxon/Kruskal-Wallis tests (rank sums). (a) ${ }^{A} P<0.05$ at day 2, 3, 4, 15, and 16. (b) ${ }^{A} P<0.05$ at day 15 and 16 .

brane, and the extent of cartilage damage. The reduction in synovial thickness in PEG-hirudin-treated mice at day 36 was significant (Figure 4c), but cartilage damage and inflammatory cells in the synovium, while diminished, did not reach statistical significance (Figure 4 , a and b, respectively).

Interestingly, when the histological scorings for the knee joint were considered (inflammatory cells, hyperplasia, and cartilage damage), a strong correlation with the clinical scoring of paw arthritis was found $(r=0.66$, $P=0.0013 ; r=0.7, P<0.0001$; and $r=0.7, P<0.0001$, respectively), showing that clinical scoring reflects the histological severity of arthritis.

Effect of PEG-hirudin treatment on fibrin deposition in arthritic joints

We wanted next to test the hypothesis that the antiinflammatory effects of PEG-hirudin in arthritic joints may be accounted for, at least in part, by decreased intra-articular fibrin deposition. Fibrin content in knee and paw joints was analyzed by fibrin immunohistochemistry in animals that had received the preventive treatment (Figure 1, b and d, for knee joints and Figure $1, \mathrm{f}$ and $\mathrm{h}$, for paw joints). The amount of fibrin in the PEG-hirudin-treated mice was less important (Figure $1, \mathrm{~d}$ and $\mathrm{h}$ ). Based on immunohistological scoring, PEG-hirudin-treated mice showed a significantly reduced level of fibrin in their knee joints compared with the control group (Figure 4d).

Correlation between fibrin deposition and clinical and histological parameters of CIA

Analysis of intra-articular fibrin deposition according to disease activity, as evaluated by clinical scoring of the paws, revealed a highly significant positive correlation $(r=0.677, P<0.0001$; Figure 5a). As expected, fibrin deposition was also correlated with plasma TAT levels, considered to be a direct indicator of thrombin generation (Figure $5 \mathrm{~b}$ ). Finally, we also found strong positive correlations between intra-articular fibrin scores and some histological features of CIA knees (inflammatory cells, hyperplasia, and cartilage damage, Figure 5, c, d, and e, respectively).

Effect of PEG-hirudin treatment on synovial cytokine mRNA expression

To obtain insight into the potential molecular mechanisms involved in the amelioration of CIA by PEGhirudin, the expression of different cytokines was investigated. Synovial mRNA levels of IL- $1 \alpha$, IL-1 $\beta$, IL-receptor antagonist, IL-12, and macrophage migration inhibitory factor were measured by RNase protection assay in the knee joints of normal mice, mice with CIA (untreated), and PEG-hirudin-treated CIA animals at day 36 (Figure 6a). As expected, synovial mRNA levels of all the cytokines analyzed were upregulated in arthritic mice compared with nonarthritic mice, with IL-1 $\beta$ exhibiting the highest mRNA level. Preventive treatment with PEG-hirudin for 16 days, as described earlier, resulted in a decrease of all the cytokine mRNA levels assayed. PhosphorImager analysis was performed to quantify mRNA expression (Figure 6b). After normalization with GAPDH, a significant downregulation of IL-1 $\beta$ and IL-12p35 mRNA $(P<0.05$ and $P<0.01$, respectively) after PEGhirudin treatment was found.

\section{Effect of PEG-hirudin treatment on synovial PAR-1 mRNA levels}

Because thrombin has been shown to increase thrombin receptor (PAR-1) expression, an additional mechanism by which PEG-hirudin could exert its effect could be by downmodulating synovial PAR-1 expression dur-
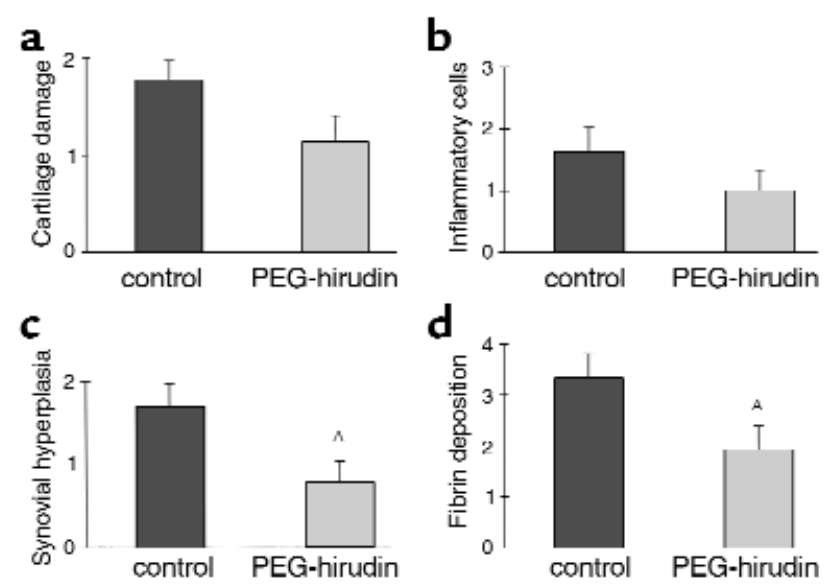

\section{Figure 4}

Histological features of arthritic knee joints. Knee histologies from control $(n=13)$ and PEG-hirudin-treated $(n=13)$ mice were scored for cartilage damage (a), inflammatory cells (b), and synovial thickness (c) using an arbitrary four-point (0-3) scale. Fibrin deposition (d), as evidenced by fibrin immunohistochemistry, was scored in the synovial membrane using an arbitrary seven-point (0-6) scale. The results are expressed as mean + SEM. Statistical significance was tested by Wilcoxon/Kruskal-Wallis tests (rank sums). ${ }^{A} P<0.05$ was considered significant. 

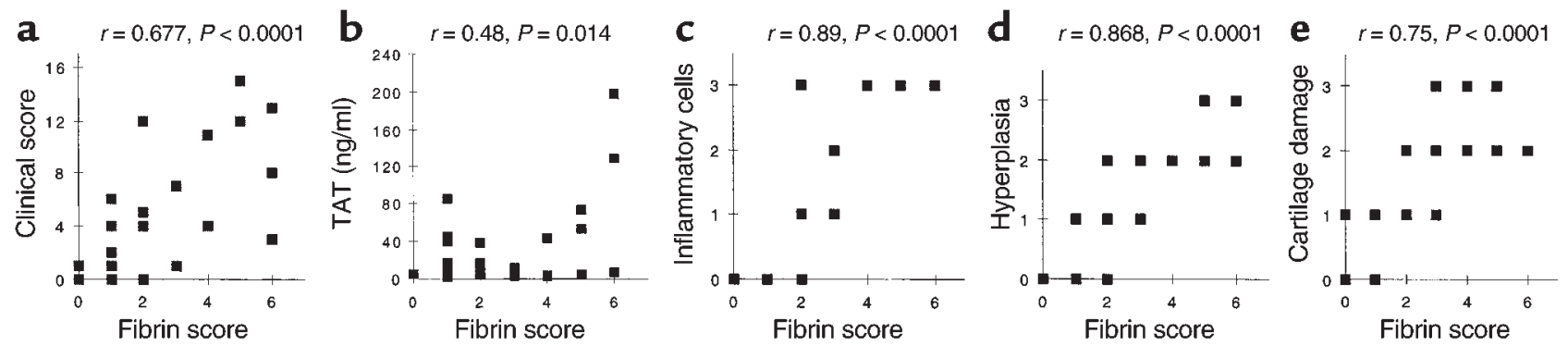

Figure 5

Correlations between fibrin staining and clinical and histological scoring. Fibrin deposition, as scored in Figure 4 was correlated to clinical score of paws (a), plasma TAT levels (b), inflammatory cells (c), synovial hyperplasia (d), and cartilage damage (e) at day 36 after immunization. $P<0.05$ was considered significant.

ing CIA. Quantification of synovial PAR-1 mRNA levels from naive or placebo- and PEG-hirudin-treated arthritic mice showed a significant downregulation of PAR-1 mRNA levels in PEG-hirudin-treated CIA mice, reaching levels found in naive mice (Figure 7).

Anticollagen Ab levels in PEG-hirudin-treated and control mice

To verify that PEG-hirudin-treated mice generated an adequate immune response against type II collagen, total anticollagen IgG levels were measured by ELISA at the end of the anticoagulation therapy (day 36). No significant difference was observed in anticollagen IgG levels between untreated and PEG-hirudin-treated mice (untreated mice: $1489.29+382.36 \mathrm{ng} / \mathrm{ml}$; treated mice: $2276.03+756.92 \mathrm{ng} / \mathrm{ml} ; n=13$ in each group).

\section{Discussion}

Evidence of activation of the coagulation pathway during inflammation raises the possibility that there is a complex interplay between the two processes that may serve to exacerbate inflammation, no matter the triggering cause, leading to chronic inflammatory changes. In the present study, we have shown that substantial fibrin deposits were present in arthritic joints of mice with CIA, and we have also provided evidence of ongoing coagulation activity, characterized by increased levels of TAT complexes in CIA mice. These results corroborate our previous findings made in the AIA model $(16,29,30)$. Thus, these two experimental models of arthritis recapitulate the extensive intra-articular fibrin deposition and the high levels of TAT found in synovial fluid $(3,7)$ and in the plasma $(3)$ of RA patients. a

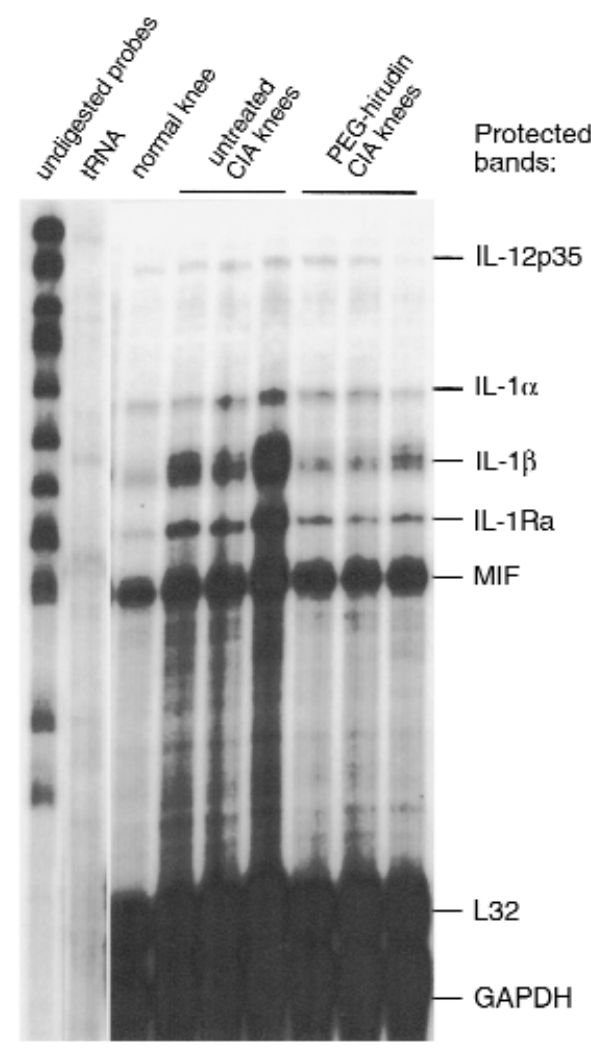

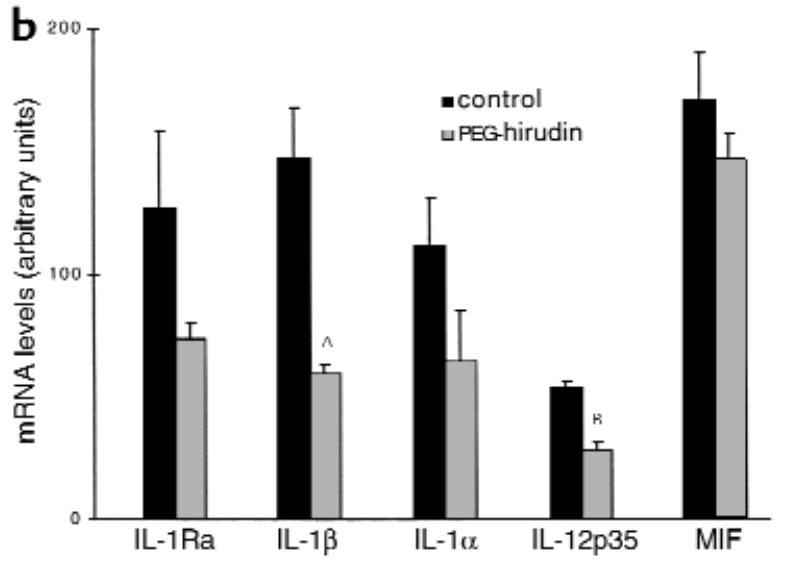

Figure 6

Synovial cytokine mRNA levels in CIA knee joints from untreated or PEGhirudin-treated mice. (a) Total RNA was prepared from knee joints of nonimmunized, nonarthritic mice (normal knee), or from arthritic CIA knee joints of untreated or PEG-hirudin-treated mice ( $1 \mathrm{mg} / \mathrm{kg} /$ day for 16 days). Expression of different cytokine mRNA was analyzed using RNase protection assay (IL-1Ra, IL-1-receptor antagonist; MIF, macrophage migration inhibitory factor). (b) Phosphorlmager analysis was performed to quantify mRNA levels of each of the studied genes. Comparison of mRNA levels is done after standardization with GAPDH mRNA and expressed in arbitrary units. The results are expressed as mean + SEM (three mice per group). ${ }^{A} P<0.05 ;{ }^{B} P<0.01$. 


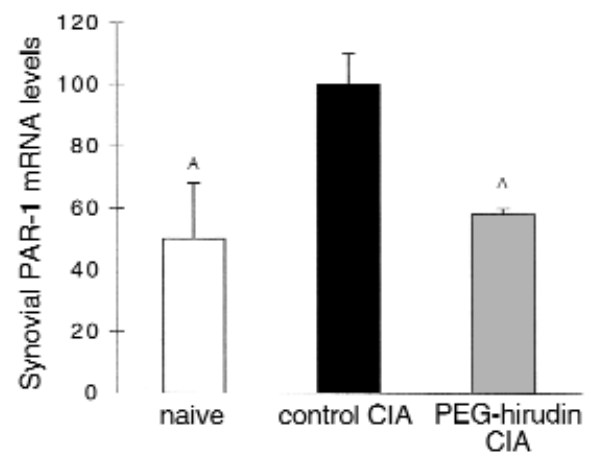

\section{Figure 7}

Synovial PAR-1 mRNA levels in CIA knee joints from untreated or PEG-hirudin-treated mice. Total RNA was prepared from dissected knee joints of nonimmunized, nonarthritic mice (naive knee) or from arthritic CIA knee joints of untreated (control CIA) or PEGhirudin-treated mice ( $1 \mathrm{mg} / \mathrm{kg} /$ day for 16 days). PAR- 1 mRNA was studied using RNase protection assay and Phosphorlmager analysis. Comparison of mRNA levels is done after standardization with GAPDH mRNA. The results are expressed as percentage of control $\mathrm{CIA}$ mice (mean + SEM, three to four mice per group). ${ }^{A} P<0.05$

In CIA, we demonstrated that besides thrombin activation, there is increased expression of PAR- $1 \mathrm{mRNA}$ in the inflamed joint, which can result from increased thrombin generation during CIA and subsequent thrombin upmodulation of PAR-1 gene expression (17, $31)$. Upregulation of PAR-1 mRNA has also been observed in human crescentic glomerulonephritis (32). In this latter case this upmodulation was associated to downregulation of PAR-1 antigen, consistent with the activation and internalization of PAR-1 that occurs after thrombin cleavage (20).

Fibrin formation and deposition is a hallmark of inflammation and we have shown previously in an experimental model of RA that intra-articular fibrin contributes to sustain synovial inflammation (16). Besides fibrin formation, another source of potential interaction between coagulation and inflammation is receptor-ligand interactions between coagulation factors and their receptors, such as for factor Xa and thrombin, that may amplify inflammation (10). As thrombin also regulates its own generation by activating factor V, VIII, IX, and X (via factor IXa), strategies that inhibit thrombin may therefore lead to decreased factor Xa and thrombin generation (33) and be therapeutic in arthritis.

PEG-hirudin was chosen over other specific thrombin inhibitors that have been described, such as argatroban or lepirudin (recombinant hirudin), because of its long duration of action and simple mode of administration. Indeed, we found that a single subcutaneous dose of 0.1 or $1 \mathrm{mg} / \mathrm{kg}$ of PEG-hirudin had an anticoagulant effect, indicated by a prolongation of the TT, even after 20 hours. These findings are consistent with the reported pharmacokinetics of PEG-hirudin (34). When coagulation times were measured at the end of the treatment phase, we noted significantly increased TT and aPTT.
These results demonstrate the efficacy by which PEGhirudin inhibits thrombin-mediated activation of the coagulation pathway and may also serve to reduce the amount of factor Xa that is generated. The PT, on the other hand, was not prolonged. An explanation for this latter observation could be that in vitro, the amount of thrombin generated during the PT assay was much greater than that generated in the TT and aPTT assays, such that the plasma concentration of PEG-hirudin in the samples was insufficient to neutralize the action of thrombin. Finally, we found that the plasma TAT-complex levels dramatically decreased when mice were treated with PEG-hirudin. Two explanations can be evoked, which are not mutually exclusive. Diminished TAT levels in treated mice could result from the inability of PEG-hirudin-inactivated thrombin to bind to antithrombin. On the other hand, treated mice may have less thrombin generation as the positive feedback on thrombin formation is being abolished.

PEG-hirudin exerted both a preventive effect and curative effect in CIA. In the preventive study, PEG-hirudin was started just before the first appearance of clinical arthritis at day 20 and continued through to day 36 . Both incidence and severity were reduced in the treated mice, and the number of affected paws was also significantly different between the two groups. The effects of PEG-hirudin on arthritis severity were reproduced using as a control, inactivated PEG-hirudin, produced essentially as described before (35), instead of PBS, emphasizing thus the specificity of active PEG-hirudin in this inhibition (results not shown). A curative effect was observed on established disease when treatment was started at the onset of clinical arthritis and continued for a total of 16 days. A significant attenuation of disease progression was observed in treated animals at the earliest times and at day 15 and 16, with a reduced change in clinical score and a reduction in number of affected paws. Moreover, though not statistically significant, a similar trend in reduced clinical scoring was observed throughout the duration of the experiment. In both treatment regimes, the effect of therapy was observed early on, with only a delayed effect on affected paws seen in the curative group.

Histological confirmation of the clinical effect was analyzed in knee joints of animals that were in the preventive study only. A significant effect was observed on synovial hyperplasia, but not on inflammatory cell infiltrate or cartilage damage, though both latter parameters were reduced in the PEG-hirudin-treated group. The histological assessment was performed on knee joints rather than paws, because the scoring system used has been extensively validated in our studies on the antigen-induced model of arthritis. Furthermore, we observed a strong correlation between histological scores and clinical score, indicating that such a scoring method does reflect changes in arthritis in the mice. The results of the cytokine mRNA assays further confirmed the reduction of inflammation in PEGhirudin-treated animals. Although only a selected sam- 
ple of cytokines were analyzed, the results are in agreement with those obtained when a broader range of cytokines were studied in CIA by Thornton et al. (36), who observed a striking enhancement of IL- $1 \beta$ and TNF- $\alpha$ RNA levels at day 35 of CIA.

Apart from its procoagulant role and its effects on target cells via the proteolytic activation of PAR-1, PAR-3, and PAR-4, thrombin exhibits chemotactic and mitogenic activity due to peptide sequences lying outside its catalytic domain (37). Since hirudin (and its derivative PEG-hirudin) can bind both to the catalytic site and the anion-binding exosite of thrombin (38), we speculate that hirudin may exert a beneficial effect in the CIA model by at least three mechanisms. First, by inhibition of thrombin procoagulant activity, leading to inhibition of $\mathrm{Xa}$ and thrombin formation, and ultimately to decreased fibrin formation; second, by inhibiting activation of the PAR- 1 receptor; and third by blocking the exosite mitogenic/chemotactic peptide domain. Concerning the first mechanism, fibrin immunohistochemistry showed a significant reduction of fibrin deposition upon PEG-hirudin treatment. Most importantly, we observed a positive correlation between clinical and histological scorings and intraarticular fibrin staining. These findings indicate that reduction of fibrin formation can attenuate synovial inflammation and corroborate our earlier findings obtained with defibrinogenated mice (16) and with fibrinogen-deficient mice (N. Busso, unpublished results), although in these latter cases the effects were much less pronounced. Recently, using a model of transplantable tumor cell lines, it has been shown that the development of lung metastasis was diminished in fibrinogen-deficient mice (39). Interestingly, hirudin further diminished the metastatic potential in these mice. In this latter case, as in our study on arthritis, it can be concluded that the thrombin inhibitor acts by at least one mechanism that is independent of fibrin(ogen). The second pathway, through which PEG-hirudin may exert its effect, is by inhibition of thrombin-receptor activation. Consistent with this hypothesis, we found that PEG-hirudin treatment of CIA mice led to decreased levels of synovial PAR-1 mRNA. Various in vitro studies have shown that signaling via PAR-1 leads to fibroblast and endothelial cell proliferation and activation as well as to activation of hematopoietic cells (monocytes, neutrophils, and lymphocytes), suggesting strongly that the PAR-1 signaling pathway is proinflammatory (17-19). Direct evidence for proinflammatory, PAR-1-dependent effects in vivo was provided by a recent study, using a murine model of immune complex-mediated glomerulonephritis (40). In this study, renal injury was attenuated by hirudin treatment, and this effect could be mimicked by PAR-1 deficiency. It remains to be seen whether the other thrombin receptors PAR-3 and PAR-4 may also mediate inflammatory changes. The availability of PAR-1-deficient mice (22) will allow a more precise investigation into these cellular actions of thrombin in vivo during experimental arthritis. The third pathway, by blockade of the chemotactic and mitogenic properties of thrombin, could be advocated if the phenotype of PAR-1-deficient mice will not recapitulate the effects of PEG-hirudin.

Our results suggest that the coagulation pathway, in particular thrombin-mediated coagulation and signaling events, may be a target for therapy in joint inflammation. Although hirudin was the only molecule studied in this series of experiments, other specific inhibitors of thrombin's actions will probably have a similarly beneficial effect (41). Such an approach may be a useful adjunct to established approaches in the treatment of the immune and cytokine-mediated pathways of arthritis.

\section{Acknowledgments}

We thank the hemostasis laboratory of the Centre Hospitalier Universitaire Vaudois for their help in clotting time measurements. We are indebted to E. Kolodziesczyk and E. Grouzmann for their generous help. This work was supported by a grant from the Fonds National Suisse de la Recherche Scientifique (32-56710.99), the Jean and Linette Warnery Foundation, and the Böni Foundation. I. Marty was a recipient of a fellowship from the Ligue Vaudoise Contre le Rhumatisme. G. Kirdaite was a recipient of a Scientific Exchange Fellowship of the Swiss confederation. C.B. Sledge, editors. W.B. Saunders Co. Philadelphia, Pennsylvania, USA. 851-897.

2. Feldmann, M., Brennan, F.M., and Maini, R.N. 1996. Rheumatoid arthritis. Cell. 85:307-310.

3. Camerer, E., Kolsto, A.B., and Prydz, H. 1996. Cell biology of tissue factor, the principal initiator of blood coagulation. Thromb. Res. 81:1-4.

4. Weinberg, J.B., Pippen, A.M., and Greenberg, C.S. 1991. Extravascular fibrin formation and dissolution in synovial tissue of patients with osteoarthritis and rheumatoid arthritis. Arthritis Rheum. 34:996-1005.

5. Zacharski, L.R., et al. 1992. Pathways of coagulation activation in situ in rheumatoid synovial tissue. Clin. Immunol. Immunopathol. 63:155-162.

6. Carmassi, F., De Negri, F., Morale, M., Puccetti, R., and Chung, S.I. 1994. Elastase- and plasmin-mediated fibrinolysis in rheumatoid arthritis. Int. J. Tissue React. 16:83-93.

7. Nakano, S., Ikata, T., Kinoshita, I., Kanematsu, J., and Yasuko, S. 1999. Characteristics of the protease activity in synovial fluid from patients with rheumatoid arthritis and osteoarthritis. Clin. Exp. Rheumatol. 17:161-170.

8. Clemmensen, I., Holund, B., and Andersen, R.B. 1983. Fibrin and fibronectin in rheumatoid synovial membrane and rheumatoid synovial fluid. Arthritis Rheum. 26:479-485

9. Jasani, M.K. 1978. Fibrin: metabolism, immunopathogenesis and significance in rheumatoid arthritis. In Immunopathogenesis of rheumatoid arthritis. G.S. Panayi and P.M. Johnsons, editors. Red Books. Surrey, United Kingdom. 137-146.

10. Cirino, G., et al. 1996. Thrombin functions as an inflammatory mediator through activation of its receptor. J. Exp. Med. 183:821-827.

11. Dvorak, H.N., Senger, D.R., Dvorak, A.M., Harvey, V.S., and McDonagh, J. 1985. Regulation of extravascular coagulation by microvascular permeability. Science. 227:1059-1061.

12. Senior, R.M., Skogen, W.F., Griffin,G.L., and Wilner, G.D. 1986. Effects of fibrinogen derivatives upon the inflammatory response. Studies with human fibrinopeptide. B. J. Clin. Invest. 77:1014-1019.

13. Perez, R.L., and Roman, J. 1995. Fibrin enhances the expression of IL1 beta by human peripheral blood mononuclear cells. Implications in pulmonary inflammation. J. Immunol. 154:1879-1887.

14. Robson, S.C., Shephard, E.G., and Kirsch, R.E. 1994. Fibrin degradation product $\mathrm{D}$-dimer induces the synthesis and release of biologically active IL- 1 beta, IL- 6 and plasminogen activator inhibitors from 
monocytes in vitro. Br.J. Haematol. 86:322-326.

15. Qi, J., and Kreutzer, D.L. 1995. Fibrin activation of vascular endothelial cells. Induction of IL-8 expression. J. Immunol. 155:867-876.

16. Busso, N., et al. 1998. Exacerbation of antigen-induced arthritis in urokinase-deficient mice. J. Clin. Invest. 102:41-50.

17. Shin, H., et al. 1995. Thrombin receptor-mediated synovial proliferation in patients with rheumatoid arthritis. Clin. Immunol. Immunopathol. 76:225-233.

18. Ohba, T., Takase, Y., Ohhara, M., and Kasukawa, R. 1996. Thrombin in the synovial fluid of patients with rheumatoid arthritis mediates proliferation of synovial fibroblast-like cells by induction of platelet derived growth factor. J. Rheumatol. 23:1505-1511.

19. Morris, R., Winyard, P.G., Blake, D.R., and Morris, C.J. 1994. Thrombin in inflammation and healing: relevance to rheumatoid arthritis. Ann. Rheum. Dis. 53:72-79.

20. Coughlin, S.R. 1998. Sol Sherry lecture in thrombosis: how thrombin 'talks' to cells: molecular mechanisms and roles in vivo. Arterioscler. Thromb. Vasc. Biol. 18:514-518.

21. Morris, R., Winyard, P.G., Brass, L.F., Blake, D.R., and Morris, C.J. 1996. Thrombin receptor expression in rheumatoid and osteoarthritic synovial tissue. Ann. Rheum. Dis. 55:841-843.

22. Vu, T.-K.H., Hung, D.T., Wheaton, V.I., and Coughlin, S.R. 1991. Molecular cloning of a functional thrombin receptor reveals a novel proteolytic mechanism of receptor activation. Cell. 64:1057-1068.

23. Markwardt, F. 1994. The development of hirudin as an antithrombotic drug. Thromb. Res. 74:1-23.

24. Markwardt, F., Nowak, G., Sturzebecher, J., Griessbach, U., Walsmann, P., and Vogel, G. 1984. Pharmacokinetics and anticoagulant effect of hirudin in man. Thromb. Haemost. 52:160-163.

25. Rubsamen, K., Hornberger, W., Laux, V., Schwarz, M., and Schweden, J. 1993. Antithrombotic efficacy of the polyethylenglycol-coupled hirudin mutein LU 57291 in experimentally induced venous and arterial thrombosis. Thromb. Haemost. 69:2717. (Abstr.)

26. Fareed, J., Walenga, J.M., Hoppenstedt, D., Iobal, O., and Rubsamen, K. 1991. Comparative pharmacodynamics of recombinant hirudin and its PEG-coupled derivatives in primates. Thromb. Haemost. 65:1286. (Abstr.)

27. Courtenay, J.S., Dallman, M.J., Dayan, A.D., Martin, A., and Mosedale, B. 1980. Immunisation against heterologous type II collagen induces arthritis in mice. Nature. 283:666-668.

28. Wooley, P.H., Luthra, H.S., Krco, C.J., Stuart, J.M., and David, C.S 1984. Type II collagen-induced arthritis in mice. II. Passive transfer and suppression by intravenous injection of anti-type II collagen antibody or free native type II collagen. Arthritis Rheum. 27:1010-1017.

29. Varisco, P.A., et al. 2000. Effect of thrombin inhibition on synovial inflammation in antigen-induced arthritis. Ann. Rheum. Dis. 59:781-787.

30. Salvi, R., Péclat, V., So, A., and Busso, N. 2000. Enhanced expression of genes involved in coagulation and fibrinolysis in murine arthritis. Artbritis Res. 2:504-512.

31. Ellis, C.A., et al. 1999. Thrombin induces proteinase-activated receptor-1 gene expression in endothelial cells via activation of Gi-linked Ras/mitogen-activated protein kinase pathway. J. Biol. Chem. 274:13718-13727.

32. Xu, Y., et al. 1995. Constitutive expression and modulation of the functional thrombin receptor in the human kidney. Am. J. Pathol, 146:101-110

33. Ofosu, F.A., Liu, L., and Freedman, J. 1996. Control mechanisms in thrombin generation. Semin. Thromb. Hemost. 22:303-308.

34. Humphries, J., et al. 1997. High and constant plasma levels of tissue plasminogen activator and PEG-hirudin can be achieved by subcutaneous delivery. Thromb. Res. 87:123-129.

35. Chang, J.-Y. 1991. Stability of hirudin, a thrombin-specific inhibitor. The structure of alkaline-inactivated hirudin. J. Biol. Chem. 266:10839-10843.

36. Thornton, S., Duwel, L., Boivin, G., Ma, Y., and Hirsch, R. 1999. Association of the course of collagen-induced arthritis with distinct patterns of cytokine and chemokine messenger RNA expression. Arthritis Rheum. 42:1109-1118.

37. Bar-Shavit, R., Kahn, A., Wilner, G.D., and Fenton, J.W. 1983. Monocyte chemotaxis: stimulation by specific exosite region in thrombin. Science. 220:728-731.

38. Maraganore, J.M., Bourdon, P., Jablonski, J., Ramachandran, K.L., and Fenton, J.W., II. 1990. Design and characterization of hirulogs: a novel class of bivalent peptide inhibitors of thrombin. Biochemistry. 29:7095-7101.

39. Palumbo, J.S., et al. 2000. Fibrinogen is an important determinant of the metastatic potential of circulating tumor cells. Blood. 96:3302-3309.

40. Cunningham, M.A., Rondeau, E., Chen, X., Coughlin, S.R., and Holdsworth, S.R. 2000. Tipping PG. Thrombin-dependent, cell-mediated renal inflammation in crescentic glomerulonephritis. J. Exp. Med. 191:455-462.

41. Fareed, J., et al. 1999. Antithrombin agents as anticoagulants and antithrombotics: implications in drug development. Semin. Hematol. 36:42-56 